\title{
Coexistência de diabetes não influencia a hipotensão pós-exercício em indivíduos com hipertensão
}

\author{
Coexistence of diabetes does not influence post-exercise hypotension in \\ individuals with hypertension
}

\author{
Alessandro Domingues Heubela, Ingrid Rita Gonçalves ${ }^{b}$, Helen Cristina Tiemi Iwamotoc, Camila Gimenes ${ }^{\mathrm{d}}$, \\ Bruna Varanda Pessoae, Bruno Martinellif, Eduardo Aguilar Arca ${ }^{f}$, Silvia Regina Barriled \\ a Fisioterapeuta. Mestrando em Fisioterapia pela Universidade Federal de São Carlos (UFSCar), São Carlos, São Paulo, Brasil. \\ Fisioterapeuta graduada pela Universidade do Sagrado Coração, Bauru, Brasil. \\ c Fisioterapeuta. Mestre em Fisioterapia pela Universidade do Sagrado Coração, Bauru, Brasil. \\ a Fisioterapeuta. Doutora em Fisiopatologia em Clínica Médica pela Universidade Estadual Paulista Júlio de Mesquita Filho. Professora da Universidade do Sagrado Coração, \\ Bauru, Brasil. \\ e Fisioterapeuta. Doutora em Fisioterapia pela UFSCar. Professora da Universidade do Sagrado Coração, Bauru, Brasil. \\ Fisioterapeuta. Doutor em Fisiopatologia em Clínica Médica pela Universidade Estadual Paulista Júlio de Mesquita Filho. Professor na Universidade do Sagrado Coração, \\ Bauru, Brasil. \\ Fontes de financiamento: Bolsa de iniciação científica pelo Conselho Nacional de Desenvolvimento Científico e Tecnológico (CNPq).
}

RESUMO

ABSTRACT
Introdução: A hipertensão arterial sistêmica (HAS) e a diabetes mellitus tipo 2 (DM2) são patologias que frequentemente coexistem. A hipotensão pós-exercício (HPE) é bem documentada em indivíduos com HAS, porém não é certo se ocorre na coexistência de DM2.

Objetivos: Investigar se a coexistência de DM2 influencia no efeito hipotensor pós-exercício aeróbio em indivíduos com HAS.

Materiais e Métodos: Foram incluídos 33 indivíduos, distribuídos em dois grupos: Grupo HAS ( $n=17$ ) e Grupo HAS+DM2 $(n=16)$. Ambos submetidos às sessões exercício e controle. O protocolo de exercício consistiu em 10 minutos de aquecimento, 40 minutos de exercício aeróbio moderado em cicloergômetro e 10 minutos de alongamentos. No pré e pós-exercício (sete horas subsequentes), foram avaliados quanto à pressão arterial (PA), frequência cardíaca (FC) e duplo produto (DP). A análise estatística foi realizada pelo teste t independente e ANOVA de medidas repetidas, seguido do post hoc de Tukey.

Resultados: Ambos os grupos apresentaram redução $(p<0,05)$ dos parâmetros hemodinâmicos após o exercício, com duração de até quatro horas. No Grupo HAS, observamos diminuição dos valores de PA sistólica (até -11 mmHg), PA diastólica (até $-7 \mathrm{mmHg}$ ), FC (até -5bpm) e DP (até -1240 bpm.mmHg). No Grupo HAS+DM2, verificamos redução da PA sistólica (até $-9 \mathrm{mmHg}$ ), FC (até -5 bpm) e DP (até -1206 bpm.mmHg).

Conclusão: $O$ exercício aeróbio moderado promoveu redução de parâmetros hemodinâmicos em indivíduos com HAS isolada e na coexistência de DM2. No entanto, verificamos que a magnitude da HPE não foi alterada na coexistência de DM2.

Palavras-chave: hipertensão arterial; diabetes mellitus tipo 2; exercício físico; hipotensão pós-exercício. documented in individuals with hypertension, but it is not certain whether it also occurs in the coexistence of type 2 diabetes mellitus. Objectives: To investigate whether the coexistence of type 2 diabetes mellitus influences the hypotensive effect after aerobic exercise in individuals with hypertension.

Materials and Methods: Thirty-three individuals were divided into two groups: Hypertension group $(n=17)$ and Hypertension + type 2 diabetes mellitus group $(n=16)$. Both groups performed exercise and control sessions. The exercise protocol consisted of 10 minutes of warm-up, 40 minutes of moderate aerobic exercise on a cycle ergometer and 10 minutes of stretching. Blood pressure, heart rate and double product were evaluated in pre- and post-exercise (until seven hours later). Statistical analysis was performed by independent $t$ test and repeated measures ANOVA followed by Tukey's post hoc test.

Results: Both groups had a reduction $(p<0.05)$ in hemodynamic parameters after exercise, lasting up to four hours. In the Hypertension group, we observed a decrease in values of systolic blood pressure (up to $-11 \mathrm{mmHg}$ ), diastolic blood pressure (up to $-7 \mathrm{mmHg}$ ), heart rate (up to -5 bpm) and double product (up to $-1240 \mathrm{bpm} . \mathrm{mmHg}$ ). In the Hypertension + type 2 diabetes mellitus group, we observed reduction of systolic blood pressure (up to $-9 \mathrm{mmHg}$ ), heart rate (up to $-5 \mathrm{bpm}$ ) and double product (up to $-1206 \mathrm{bpm} . \mathrm{mmHg}$ ).

Conclusion: The moderate aerobic exercise promoted reduction of hemodynamic parameters in individuals with isolated hypertension and hypertension plus type 2 diabetes mellitus. However, we verified that the magnitude of post-exercise hypotension was not altered with the coexistence of type 2 diabetes mellitus.

Keywords: hypertension; type 2 diabetes mellitus; physical exercise; post-exercise hypotension. 


\section{INTRODUÇÃO}

A hipertensão arterial sistêmica (HAS) e a diabetes mellitus tipo 2 (DM2) são patologias que frequentemente coexistem ${ }^{1}$. As complicações decorrentes do mau controle pressórico são responsáveis por 7,6 milhões de mortes no mundo $^{2}$. No Brasil, estima-se que a prevalência de HAS tenha diminuído $6 \%$ nas últimas três décadas, porém ainda está presente em $30 \%$ da população ${ }^{3}$.

Dentre os principais tratamentos não-farmacológicos da HAS, o exercício físico aeróbio é considerado fundamental, uma vez que pode ocasionar a redução imediata e sustentada da pressão arterial (PA), em um fenômeno conhecido como hipotensão pós-exercício (HPE) ${ }^{4}$. Os mecanismos envolvidos no desencadeamento da HPE ainda não estão totalmente esclarecidos, porém acredita-se que o sistema nervoso autonômico e substâncias vasodilatadoras exerçam papelchave na redução da resistência vascular periférica 5 .

Embora o efeito hipotensor do exercício aeróbio em indivíduos com HAS esteja bem descrito ${ }^{6-9}$, poucas são as evidências que estudaram se o mesmo também ocorre na coexistência da DM2, visto que a DM2 por si só está associada a diversos déficits fisiológicos, tais como aumento da rigidez arterial, prejuízos na função endotelial e disfunção autonômica ${ }^{10,11}$. Essas condições, associadas às outras comorbidades, como a HAS, poderiam influenciar no efeito hipotensor após o exercício ${ }^{12}$. Assim, o presente estudo teve como objetivo investigar se a coexistência da DM2 influencia no efeito hipotensor pós-exercício aeróbio em indivíduos com HAS. A nossa hipótese principal é que a HPE seja mais atenuada na coexistência DM2 e HAS quando comparada a HAS isolada.

\section{MATERIAIS E MÉTODOS}

Trata-se de um ensaio clínico, não controlado e não randomizado, com delineamento cruzado, no qual a amostra foi selecionada por conveniência, na Clínica de Fisioterapia da Universidade do Sagrado Coração (USC), Bauru, São Paulo. O presente estudo foi aprovado pelo Comitê de Ética em Pesquisa da Pró-Reitoria de Pesquisa e Pós-Graduação da USC (protocolo no 005). Os voluntários que concordaram em participar do estudo, após orientação detalhada sobre os objetivos e procedimentos, assinaram o Termo de Consentimento Livre e Esclarecido.

A amostra total consistiu em 33 indivíduos, de ambos os sexos. Inicialmente, houve o convite para os indivíduos participarem da pesquisa, antes de iniciarem o atendimento na clínica de Fisioterapia, no estágio de Fisioterapia em Cardiologia. Foram incluídos os indivíduos que atenderam aos seguintes critérios: 1) não praticantes de programa de exercício físico há pelo menos três meses; 2) com atestado médico negativo para cardiopatias; 3) ausência de patologias neurológicas, musculoesqueléticas e/ou cardiovasculares que impedissem a prática de exercício. Após a triagem inicial e assinatura do termo de consentimento livre e esclarecido, os indivíduos foram alocados em dois grupos: grupo hipertensão arterial sistêmica (HAS, $n=17$ ) e grupo hipertensão arterial sistêmica associada a diabetes mellitus tipo 2 (HAS+DM2, $\mathrm{n}=16)$.

\section{Avaliações iniciais}

A anamnese clínica foi realizada seguindo questionário padronizado, no qual constavam informações pessoais, sobre a história da moléstia e medicamentos utilizados. A massa corporal foi obtida por meio de uma balança digital (Filizola ${ }^{\circledR}$, São Paulo) e a estatura medida por um estadiômetro (WCS ${ }^{\circledR}$, Brasil). O índice de massa corporal (IMC) foi calculado por meio da equação $I M C=$ massa corporal/estatura ${ }^{2}$. A circunferência abdominal foi mensurada com auxílio de uma trena antropométrica (Cescorf ${ }^{\circledR}$, Brasil) no nível da cicatriz umbilical. As medidas de pressão arterial (PA) e frequência cardíaca (FC) foram obtidas em repouso, com auxílio de aparelho de pressão semiautomático (Omron ${ }^{\circledR}$, Brasil), devidamente calibrado, e seguindo as recomendações da Sociedade Brasileira de Cardiologia ${ }^{13}$. O duplo produto (DP) foi calculado pela fórmula $\mathrm{DP}=\mathrm{PA}$ sistólica $\times \mathrm{FC}^{14}$.

\section{Avaliação hemodinâmica}

As medidas de PA e FC foram obtidas por meio do mesmo aparelho de pressão semiautomático (Omron ${ }^{\circledR}$, Brasil). Todas as medidas foram verificadas em repouso, em sessões controle e exercício, separadas por intervalo mínimo de 72 horas. Em ambas as sessões, os indivíduos foram avaliados no momento inicial (pré) e em sete momentos pós-intervenção (M1 a 7), com intervalos de 60 minutos. Em todos os momentos, a medida da pressão arterial foi realizada pelo protocolo de automedida da pressão arterial (AMPA), no qual consiste em uma modalidade de medição realizada pelo próprio indivíduo e que se correlaciona à medida ambulatorial da pressão arterial ${ }^{15}$. Nos dias de medida, os indivíduos foram instruídos previamente quanto à utilização do aparelho e receberam as orientações para a medida da PA em domicílio, de acordo com as recomendações das Sociedades Brasileiras de Cardiologia, Hipertensão e Nefrologia ${ }^{16}$.

\section{Exercício físico}

Ambos os grupos foram submetidos às sessões exercício e controle, iniciadas entre 13 e 14 horas. O protocolo de exercício físico consistiu em 40 minutos de exercício aeróbio contínuo em cicloergômetro (Kettler Stratos ${ }^{\circledR}$, Alemanha), 
precedido por 10 minutos de aquecimento no mesmo equipamento e seguido de 10 minutos de alongamentos. A intensidade do aquecimento e do exercício foi monitorada por cardiofrequencímetro (Polar ${ }^{\circledR}$, Finlândia) e mantida inferior a $60 \%$ (leve) e $64-76 \%$ (moderada) da $\mathrm{FC}$ máxima $\left(\mathrm{FC}_{\text {máx }}\right)$, respectivamente ${ }^{17}$. O cálculo da $\mathrm{FC}_{\text {máx }}$ foi realizado pela fórmula $\mathrm{FC}_{\text {máx }}=220-$ idade $^{18}$. Para os indivíduos que utilizavam $\beta$-bloqueadores, foi utilizada a fórmula para correção da FC de acordo com a dosagem de propranolol ou fármaco equivalente ${ }^{19}$. Na sessão controle, os indivíduos permaneceram em repouso e receberam apenas orientações sobre fatores de risco e hábitos de vida saudáveis.

\section{Análise estatística}

Os resultados deste estudo foram analisados pelo programa Statistical Package for Social Sciences para Windows, versão 20.0 (IBM ${ }^{\circledR}$, Estados Unidos). A normalidade dos dados foi verificada pelo teste de Shapiro-Wilk e as variáveis quantitativas com distribuição normal foram expressas em média \pm desvio padrão. As variáveis categóricas foram descritas em frequência relativa e comparadas pelo teste de qui-quadrado.

A análise das variáveis dependentes (PAS, PAD, FC e DP) no pós-exercício ou controle foi realizada pela ANOVA de medidas repetidas, seguido do teste post hoc de Tukey. A comparação intergrupos das variações hemodinâmicas nos momentos pós-exercício foi realizada pelo teste $t$ para amostras independentes. Em todas as análises foi adotado nível de significância de 5\%.

\section{RESULTADOS}

A Tabela 1 mostra as características demográficas, antropométricas, hemodinâmicas, bem como os medicamentos utilizados pelos indivíduos dos grupos HAS e HAS+DM2. Em relação às variáveis demográficas, antropométricas e hemodinâmicas, não foram observadas diferenças significativas entre ambos os grupos, evidenciando a homogeneidade da amostra. No que se refere aos medicamentos, a maior utilização de antidiabéticos orais pelo grupo HAS+DM2 era esperada, uma vez que os indivíduos possuíam diagnóstico de DM2 e realizavam tratamento farmacológico.

A Figura 1 mostra o comportamento hemodinâmico do Grupo HAS no dia controle e exercício. No dia exercício, observamos redução significativa $(p<0,05)$ das seguintes variáveis: PAS (M2 e M3), PAD (M1, M2 e M4), FC (M3) e DP (M2 e M3). No dia controle, verificamos aumento significativo $(p<0,05)$ da FC em M1, M2, M4 e M5, e manutenção das demais variáveis.
Tabela 1. Características demográficas, antropométricas, hemodinâmicas e medicamentos utilizados pelos grupos hipertensão (HAS) e hipertensão associado a diabetes (HAS+DM2).

\begin{tabular}{|c|c|c|c|}
\hline Características & $\underset{(n=17)}{\text { HAS }}$ & $\begin{array}{c}\text { HAS + DM2 } \\
\quad(n=16)\end{array}$ & $p$ \\
\hline Mulheres, n (\%) & $13(76,5)$ & $12(75,5)$ & 0,922 \\
\hline Idade (anos) & $65,7 \pm 12,0$ & $63,0 \pm 9,1$ & 0,483 \\
\hline Massa corporal (kg) & $70,2 \pm 13,9$ & $79,6 \pm 12,7$ & 0,052 \\
\hline IMC (kg/m²) & $27,0 \pm 5,3$ & $30,2 \pm 5,2$ & 0,082 \\
\hline $\mathrm{CA}(\mathrm{cm})$ & $97,3 \pm 13,7$ & $105,1 \pm 12,3$ & 0,094 \\
\hline PAS (mmHg) & $133,1 \pm 1,9$ & $126,9 \pm 4,3$ & 0,384 \\
\hline PAD (mmHg) & $75,2 \pm 0,2$ & $71,9 \pm 1,8$ & 0,423 \\
\hline FC (bpm) & $72,4 \pm 2,4$ & $74,3 \pm 1,6$ & 0,538 \\
\hline DP (bpm.mmHg) & $9661,8 \pm 186,9$ & $9591,1 \pm 326,2$ & 0,934 \\
\hline \multicolumn{4}{|l|}{ Antihipertensivos } \\
\hline Diuréticos, n (\%) & $5(29,4)$ & $2(12,5)$ & 0,235 \\
\hline Inibidores centrais, n (\%) & $4(23,5)$ & $1(6,2)$ & 0,166 \\
\hline Inibidores da ECA, n (\%) & $7(41,2)$ & $6(37,5)$ & 0,829 \\
\hline$\beta$-bloqueador, n (\%) & $2(11,8)$ & $1(6,2)$ & 0,582 \\
\hline $\begin{array}{l}\text { Antagonistas dos canais } \\
\text { de Ca, } \mathrm{n}(\%)\end{array}$ & $1(5,9)$ & $1(6,2)$ & 0,965 \\
\hline BRA, n (\%) & $4(23,5)$ & $6(37,5)$ & 0,383 \\
\hline Antidiabéticos orais, n (\%) & $0(0,0)$ & $14(87,5)$ & 0,000 \\
\hline Insulina, n (\%) & $0(0,0)$ & $2(12,5)$ & 0,133 \\
\hline
\end{tabular}

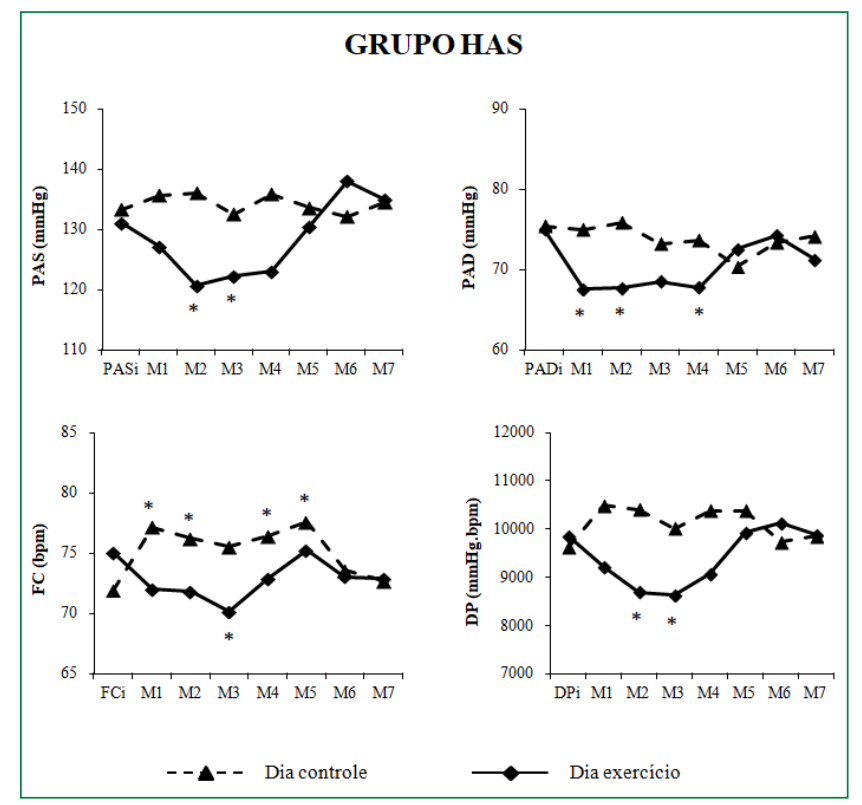

Figura 1. Grupo hipertensão arterial sistêmica (HAS): comportamento da pressão arterial sistólica (PAS), diastólica (PAD), frequência cardíaca (FC) e duplo produto (DP) no pré e nos sete momentos (M1 a M7) pós-sessão exercício ou controle. ${ }^{*} p<0,05$ quando comparado ao momento inicial (repouso) da mesma sessão. Valor de $p$ obtido pela ANOVA de medidas repetidas, seguido do teste post hoc de Tukey. 
A Figura 2 evidencia o comportamento hemodinâmico do Grupo HAS+DM2 no dia controle e exercício. No dia exercício, verificamos diminuição significativa $(p<0,05)$ das seguintes variáveis: PAS (M1 e M2), FC (M2) e DP (M1, M2 e M3). No dia controle, observamos aumento significativo $(p<0,05)$ da PAS (M1, M3, M4 e M5), FC (M1) e DP (M1, M3 e M4).

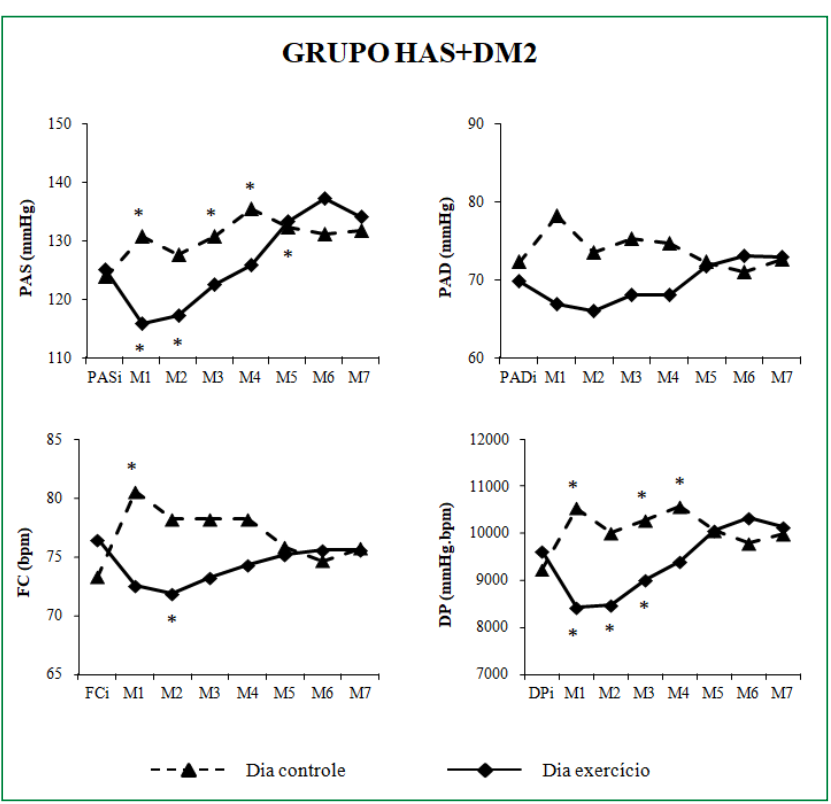

Figura 2. Grupo hipertensão arterial sistêmica (HAS) + diabetes mellitus tipo 2 (DM2): comportamento da pressão arterial sistólica (PAS), diastólica (PAD), frequência cardíaca ( $\mathrm{FC}$ ) e duplo produto (DP) no pré e nos sete momentos (M1 a M7) pós-sessão exercício ou controle. ${ }^{*} p<0,05$ quando comparado ao momento inicial (repouso) da mesma sessão. Valor de $p$ obtido pela ANOVA de medidas repetidas, seguido do teste post hoc de Tukey.

A Tabela 2 mostra a variação $(\Delta)$ dos parâmetros hemodinâmicos de ambos os grupos, nos sete momentos (M1 à 7) pós-exercício. Não observamos diferença significativa na comparação intergrupos.

\section{DISCUSSÃO}

O presente estudo objetivou verificar se a coexistência de DM2 influencia no efeito hipotensor pós-exercício aeróbio em indivíduos com HAS. A partir dos resultados encontrados, observamos que o exercício aeróbio promoveu hipotensão em ambos os grupos, porém tais efeitos foram observados até no máximo quatro horas após o exercício. Além disso, verificamos que a coexistência de DM2 não influenciou no efeito anti-hipertensivo pós-exercício aeróbio em indivíduos com HAS.

Corroborando nossos achados, o efeito hipotensor do exercício aeróbio em indivíduos com HAS é amplamente descrito em outros estudos ${ }^{6-9}$. Os mecanismos responsáveis pela redução da PA pós-exercício ainda não são completamente entendidos, porém acredita-se que a diminuição da atividade simpática e a vasodilatação periférica exerçam papel-chave nesse fenômeno ${ }^{20}$. Ademais, MacDonald ${ }^{21}$ ressalta outros possíveis mecanismos envolvidos, tais como a diminuição do débito cardíaco e a redução da resistência vascular periférica.

Em relação à duração do efeito hipotensor, no presente estudo verificamos sustentação por até quatro horas após o exercício. Em outros estudos, a duração da hipotensão pós-exercício aeróbio é variável, com amplitude de quatro a 24 horas $^{22}$. De acordo com Guidry et al. ${ }^{23}$, as diferenças na duração do efeito hipotensor podem ser justificadas pelos diferentes tipos, intensidades e volumes de exercício. Como exemplo, Eicher et al. ${ }^{24}$ verificaram que, para cada $10 \%$ de aumento no $\mathrm{VO}_{2 \text { pico, }}$ ocorre diminuição de $1,5 \mathrm{mmHg}$ e 0,6 mmHg na PA sistólica e diastólica, respectivamente, em indivíduos hipertensos submetidos à diferentes intensidades de exercício aeróbio. Nessa mesma linha, Pescatello et al. ${ }^{25}$ estudaram o efeito do exercício aeróbio moderado $\left(40 \% \mathrm{VO}_{2 \max }\right)$ e vigoroso $\left(60 \% \mathrm{VO}_{2 \max }\right)$ em homens hipertensos e constataram declínio da PA após ambas as sessões,

Tabela 2. Comparação intergrupos das variações $(\Delta)$ hemodinâmicas nos momentos pós-exercício (M1 à M7).

\begin{tabular}{|c|c|c|c|c|c|c|c|c|c|c|c|c|}
\hline & \multicolumn{3}{|c|}{$\triangle$ PAS $(\mathbf{m m H g})$} & \multicolumn{3}{|c|}{$\triangle$ PAD $(\mathbf{m m H g})$} & \multicolumn{3}{|c|}{$\Delta \mathrm{FC}(\mathrm{bpm})$} & \multicolumn{3}{|c|}{$\Delta \mathrm{DP}(\mathrm{bpm} . \mathrm{mmHg})$} \\
\hline & HAS & HAS + DM2 & $p$ & HAS & HAS+DM2 & $p$ & HAS & HAS + DM2 & $p$ & HAS & HAS+DM2 & $p$ \\
\hline M1 & $-4 \pm 14$ & $-9 \pm 13$ & 0,28 & $-7 \pm 11$ & $-3 \pm 11$ & 0,26 & $-3 \pm 7$ & $-4 \pm 9$ & 0,72 & $-643 \pm 1703$ & $-1206 \pm 1494$ & 0,32 \\
\hline M2 & $-11 \pm 15$ & $-8 \pm 12$ & 0,55 & $-7 \pm 13$ & $-4 \pm 9$ & 0,41 & $-3 \pm 8$ & $-5 \pm 11$ & 0,67 & $-1178 \pm 1648$ & $-1151 \pm 1489$ & 0,96 \\
\hline M3 & $-9 \pm 17$ & $-2 \pm 14$ & 0,25 & $-6 \pm 14$ & $-2 \pm 11$ & 0,30 & $-5 \pm 9$ & $-3 \pm 5$ & 0,53 & $-1240 \pm 1958$ & $-606 \pm 1136$ & 0,26 \\
\hline M4 & $-8 \pm 19$ & $1 \pm 11$ & 0,11 & $-7 \pm 11$ & $-2 \pm 7$ & 0,11 & $-2 \pm 11$ & $-2 \pm 5$ & 0,96 & $-791 \pm 2274$ & $-229 \pm 833$ & 0,35 \\
\hline M5 & $-1 \pm 19$ & $8 \pm 15$ & 0,14 & $-2 \pm 12$ & $2 \pm 7$ & 0,22 & $0 \pm 9$ & $-1 \pm 7$ & 0,60 & $31 \pm 2095$ & $447 \pm 1598$ & 0,55 \\
\hline M6 & $7 \pm 20$ & $12 \pm 17$ & 0,41 & $-1 \pm 13$ & $3 \pm 8$ & 0,30 & $-2 \pm 9$ & $-1 \pm 8$ & 0,72 & $264 \pm 2081$ & $722 \pm 1459$ & 0,47 \\
\hline M7 & $4 \pm 21$ & $9 \pm 12$ & 0,39 & $-4 \pm 12$ & $3 \pm 10$ & 0,08 & $-2 \pm 11$ & $-1 \pm 6$ & 0,69 & $3 \pm 2299$ & $504 \pm 1061$ & 0,43 \\
\hline
\end{tabular}

PAS: pressão arterial sistólica; PAD: pressão arterial diastólica; FC: frequência cardíaca; DP: duplo produto; HAS: hipertensão arterial sistêmica (grupo); DM2: diabetes mellitus tipo 2 (grupo). Valor de $p$ obtido pelo teste $t$ para amostras independentes. 
porém com maior magnitude na sessão moderada. Em nosso estudo, optamos por estudar o exercício aeróbio em intensidade moderada, uma vez que a mesma é inicialmente recomendada no tratamento de indivíduos hipertensos ${ }^{26,27}$.

No presente estudo, embora ambos os grupos tenham apresentado redução da PA no pós-exercício, não verificamos diferenças quanto à magnitude de variação, indicando que a coexistência de DM2 não influenciou nas alterações hemodinâmicas. Simões et al..$^{28}$ estudaram o efeito agudo do exercício aeróbio moderado ( $90 \%$ do limiar de lactato) na PA de dez indivíduos com DM2 (sendo cinco hipertensos) comparando com dez indivíduos sem DM2. Nesse mesmo estudo, os autores verificaram a ocorrência da HPE em ambos os grupos, porém com atenuação da intensidade e duração do efeito no grupo DM2. De acordo com os autores, a menor magnitude da HPE observada em indivíduos com DM2 é parcialmente explicada pela diminuição da atividade enzimática calicreína-cinina, redução da concentração de bradicinina e prejuízo na liberação de óxido nítrico ${ }^{28}$.

$\mathrm{Na}$ análise do comportamento da FC, observamos diminuição em até três horas após o exercício, com posterior tendência para o retorno aos valores basais. Tal fenômeno pode ser explicado, em parte, pelo efeito do sistema nervoso autonômico sobre o coração, sabendo que há diminuição da modulação simpática após o exercício, a depender da intensidade e volume do mesmo ${ }^{12}$. Além disso, outra possível explicação estaria relacionada ao estímulo do exercício para a produção de substâncias vasodilatadoras, tais como o óxido nítrico e a histamina ${ }^{29,30}$. Como consequência, o efeito vasodilatador resultaria em redução da resistência vascular periférica e pós-carga cardíaca, eventos que contribuiriam para diminuição do trabalho cardíaco ${ }^{31}$.

No que diz respeito ao DP, observamos redução significativa e sustentada até três horas após o exercício em ambos os grupos. De acordo com Ansari et al. ${ }^{32}$, o DP é uma medida indireta que representa o trabalho cardíaco ou o consumo de oxigênio pelo miocárdio. Em nosso trabalho, consideramos como principal hipótese para a diminuição do DP a vasodilatação ocasionada pelo exercício ${ }^{32}$, como mencionado anteriormente. Adicionalmente, considerando que o DP está associado ao risco cardiovascular ${ }^{14}$, interpretamos a redução do DP como um indicativo de melhora da saúde cardiovascular, mesmo que agudamente.

Apesar dos resultados obtidos em nosso estudo, destacamos algumas limitações. A primeira delas diz respeito ao método empregado para a verificação da PA após o exercício. Embora os indivíduos tenham sido treinados previamente quanto à automedida, ressalta-se que a mesma estaria sujeita a erros de procedimento, uma vez que o indivíduo não foi supervisionado. Por outro lado, destacamos que a AMPA pode ser considerada uma alternativa mais econômica e acessível quando comparada a MAPA. Outra limitação está relacionada ao fato de que, nas sete horas subsequentes ao exercício, não foi realizado controle da ingestão de líquidos e alimentação, bem como das atividades realizadas pelos indivíduos. Claramente, entendemos que tais ocorrências poderiam influenciar nos parâmetros hemodinâmicos avaliados. Por fim, consideramos o tamanho amostral pequeno, tornando-se necessários outros estudos para confirmação de nossos achados.

Nossos resultados sugerem que o exercício aeróbio em intensidade moderada promove hipotensão pós-exercício em indivíduos com hipertensão arterial isolada e na coexistência de diabetes mellitus tipo 2. No entanto, verificamos que a magnitude do efeito hipotensor não é alterada na coexistência de diabetes mellitus tipo 2. Futuros estudos devem buscar compreender se tal fenômeno pode ser influenciado por diferentes tipos e intensidades de exercício.

\section{AGRADECIMENTOS}

Agradecemos ao Conselho Nacional de Desenvolvimento Científico e Tecnológico (CNPq), pelo suporte financeiro proporcionado durante a execução do trabalho.

\section{REFERÊNCIAS}

1. Cheung BM, Li C. Diabetes and hypertension: is there a common metabolic pathway? Curr Atheroscler Rep. 2012;14(2):160-6. https://doi.org/10.1007/s11883-012-0227-2

2. Arima H, Barzi F, Chalmes J. Mortality patterns in hypertension. J Hypertension. 2011;29(1):S3-7. https://doi.org/10.1097/01. hjh.0000410246.59221.b1

3. Picon RV, Fuchs FD, Moreira LB, Riegel G, Fuchs SC. Trends in prevalence of hypertension in Brazil: a systematic review with metaanalysis. PLoS One. 2012;7(10):e48255. https://doi.org/10.1371/ journal.pone.0048255

4. Anunciação PG, Polito MD. A review on post-exercise hypotension in hypertensive individuals. Arq Bras Cardiol. 2011;96(5):100-9.

5. Halliwill JR. Mechanisms and clinical implications of post-exercise hypotension in humans. Exerc Sport Sci Rev. 2001;29(2):65-70.

6. Cunha F, Midgley AW, Pescatello L, Soares PP, Farinatti P. Acute hypotensive response to continuous and accumulated isocaloric aerobic bouts. Int J Sports Med. 2016;37(11):855-62. https://doi. org/10.1055/s-0042-104197

7. Brito LC, Rezende RA, Silva-Junior ND, Tinucci T, Casarini DE, Cipolla-Neto J, Forjaz CLM. Post-exercise hypotension and its mechanisms differ after morning and evening exercise: a randomized crossover study. PLoS One. 2015;10(7):1-16. https:// doi.org/10.1371/journal.pone.0132458

8. Carvalho RS, Pires CM, Junqueira GC, Freitas D, Marchi-Alves LM. Hypotensive response magnitude and duration in hypertensives: continuous and interval exercise. Arq Bras Cardiol. 2015;104(3): $234-41$. 
9. Oliveira J, Mesquita-Bastos J, Argel de Melo C, Ribeiro F. Post aerobic exercise blood pressure reduction in very old persons with hypertension. J Geriatr Phys Ther. 2016;39(1):8-13. https://doi. org/10.1519/JPT.0000000000000049

10. Christen Al, Armentano RL, Miranda A, Graf S, Santana DB, Zócalo Y, Baglivo HP, Sánchez RA. Arterial wall structure and dynamics in type 2 diabetes mellitus methodological aspects and pathophysiological findings. Curr Diabetes Rev. 2010;6(6):367-77. https://doi.org/10.2174/157339910793499146

11. Sucharita S, Bantwal G, Idiculla J, Ayyar V, Vaz M. Autonomic nervous system function in type 2 diabetes using conventional clinical autonomic tests, heart rate and blood pressure variability measures. Indian J Endocrionol Metab. 2011;15(3):198-203. https://doi.org/10.4103/2230-8210.83406

12. Asano RY, Sales MM, Browne RAV, Moraes JFVM, Coelho-Júnior J, Moraes MR, Simões HG. Acute effects of physical exercise in type 2 diabetes: a review. World J Diabetes. 2014;5(5):659-65. https:// doi.org/10.4239/wjd.v5.i5.659

13. Malachias MVB, Souza WKSB, Plavnik FL, Rodrigues CIS, Brandão AA, Neves MFT, Bortolotto LA, Franco RJS, Poli-de-Figueiredo CE, Jardim PCBV, Amodeo C, Barbosa ECD, Koch V, Gomes MAM, Paula RB, Póvoa RMS, Colombo FC, Ferreira Filho S, Miranda RD, Machado CA, Nobre F, Nogueira AR, Mion Júnior D, Kaiser S, Forjaz CLM, Almeida FA, Martim JFV, Sass N, Drager LF, Muxfeldt E, Bodanese LC, Feitosa AD, Malta D, Fuchs S, Magalhães ME, Oigman W, Moreira Filho O, Pierin AMG, Feitosa GS, Bortolotto MRFL, Magalhães LBNC, Silva ACS, Ribeiro JM, Borelli FAO, Gus M, Passarelli Júnior O, Toledo JY, Salles GF, Martins LC, Jardim TSV, Guimarães ICB, Antonello IC, Lima Júnior E, Matsudo V, Silva GV, Costa LS, Alessi A, Scala LCN, Coelho EB, Souza D, Lopes HF, Gowdak MMG, Cordeiro Júnior AC, Torloni MR, Klein MRST, Nogueira PK, Lotaif LAD, Rosito GBA, Moreno Júnior H. 7aㅡ Diretriz Brasileira de Hipertensão Arterial. Arq Bras Cardiol. 2016;107(3 supl 3):1-83.

14. White WB. Heart rate and the rate-pressure product as determinants of cardiovascular risk in patients with hypertension. Am J Hypertens. 1999;12(2):50-5. https://doi.org/10.1016/S0895-7061(98)00280-5

15. Gaborieau V, Delarche N, Gosse P. Ambulatory blood pressure monitoring versus self-measurement of blood pressure at home: correlation with target organ damage. J Hypertens. 2008;26(10): 1919-27. https://doi.org/10.1097/HJH.0b013e32830c4368

16. Sociedades Brasileiras de Cardiologia, Hipertensão e Nefrologia. $\checkmark$ Diretrizes Brasileiras de Monitoração Ambulatorial da Pressão Arterial (MAPA V) e III Diretrizes de Monitoração Residencial da Pressão Arterial (MRPA III). Arq Bras Cardiol. 2011;97(3):1-26.

17. Garber CE, Blissmer B, Deschenes MR, Franklin BA, Lamonte MJ, Lee IM, Nieman DC, Swain DP. American College of Sports Medicine position stand. Quantity and quality of exercise for developing and maintaining cardiorespiratory, musculoskeletal, and neuromotor fitness in apparently healthy adults: guidance for prescribing exercise. Med Sci Sports Exerc. 2011;43(7):1334-59. https://doi.org/10.1249/MSS.0b013e318213fefb

18. Karvonen MJ, Kentala E, Mustala O. The effects of training on heart rate: a longitudinal study. Ann Med Exp Biol Fenn. 1957;35(3): 307-15.

19. Sociedade Brasileira de Cardiologia. I Consenso Nacional de Reabilitação Cardiovascular. Arq Bras Cardiol. 1997;69(4):267-91.
20. Zafeiridis A. Mechanisms and exercise characteristics influencing postexercise hypotension. Br J Med Med Res. 2014;4(36): 5699-714. https://doi.org/10.9734/BJMMR/2014/12731

21. MacDonald JR. Potential causes, mechanisms, and implications of post exercise hypotension. J Hum Hypertens. 2002;16(4):225-36. https://doi.org/10.1038/sj.jhh.1001377

22. Cardoso Jr CG, Gomides RS, Queiroz ACC, Pinto LG, Lobo FS, Tinucci T, Mion Jr D, Forjaz CLM. Acute and chronic effects of aerobic and resistance exercise on ambulatory blood pressure. Clinics. 2010;65(3):317-25. https://doi.org/10.1590/S180759322010000300013

23. Guidry MA, Blanchard BE, Thompson PD, Maresh CM, Seip RL, Taylor AL, Pescatello LS. The influence of short and long duration on the blood pressure response to an acute bout of dynamic exercise. Am Heart J. 2006;151(6):1322.e5-12. https://doi.org/10.1016/j. ahj.2006.03.010

24. Eicher JD, Maresh CM, Tsongalis GJ, Thompson PD, Pescatello LS. The additive blood pressure effects of exercise intensity on postexercise hypotension. Am Heart J. 2010;160(3):513-20. https://doi. org/10.1016/j.ahj.2010.06.005

25. Pescatello LS, Guidry MA, Blanchard BE, Kerr A, Taylor AL, Johnson AN, Maresh CM, Rodriguez N, Thompson PD. Exercise intensity alters postexercise hypotension. J Hypertens. 2004;22(10):1881-8. https://doi.org/10.1097/00004872-200410000-00009

26. Boutcher YN, Boutcher SH. Exercise intensity and hypertension: what's new? J Hum Hypertens. 2017;31(3):157-64. https://doi. org/10.1038/jhh.2016.62

27. Pescatello LS, Franklin BA, Fagard R, Farguhar WB, Kelley GA, Ray CA. American College of Sports Medicine position stand. Exercise and hypertension. Med Sci Sports Exerc. 2004;36(3):533-53. https://doi.org/10.1249/01.MSS.0000115224.88514.3A

28. Simões HG, Asano RY, Sales MM, Browne RAV, Arsa G, MottaSantos D, Puga GM, Lima LCJ, Campbell CSG, Franco OL. Type 2 diabetes elicits lower nitric oxide, bradykinin concentration and kallikrein activity together with higher desarg9-BK and reduced post-exercise hypotension compared to non-diabetic condition. PLoS One. 2013;8(11):1-7. https://doi.org/10.1371/journal.pone. 0080348

29. Goto C, Nishioka K, Umemura T, Jitsuiki D, Sakagutchi A, Kawamura M, Chayama K, Yoshizumi M, Higashi Y. Acute moderate-intensity exercise induces vasodilation through na increase in nitric oxide. Am J Hypertens. 2007;20(8):825-30. https://doi.org/10.1016/j. amjhyper.2007.02.014

30. Halliwill JR, Buck TM, Lacewell AN, Romero SA. Postexercise hypotension and sustained postexercise vasodilatation: what happens after we exercise? Exp Physiol. 2013;98(1):7-18. https:// doi.org/10.1113/expphysiol.2011.058065

31. Romero AS, Minson CT, Halliwill JR. The cardiovascular system after exercise. J Appl Physiol. 2017;122(4):925-32. https://doi. org/10.1152/japplphysiol.00802.2016

32. Ansari $M$, Javadi $H$, Pourbehi $M$, Mogharrabi M, Rayzan M, Semnani S, Jallalat S, Amini A, Abbaszadeh M, Barekat M, Nabipour I, Assadi $M$. The association of rate pressure product (RPP) and myocardial perfusion imaging (MPI) findings: a preliminary study. Perfusion. 2012;27(3):207-13. https://doi.org/10.1177/0267659112436631 